\title{
EXACTNESS OF FORMAL ASYMPTOTIC SOLUTIONS OF A DIRICHLET PROBLEM MODELING THE STEADY STATE OF FUNCTIONALLY-GRADED MICROPERIODIC NONLINEAR RODS
}

\author{
Roberto M.S. Décio Jr. ${ }^{1}$, Leslie D. Pérez-Fernández ${ }^{2}$,Julián Bravo-Castillero ${ }^{3}$ \\ ${ }^{1}$ Programa de Pós-Graduação em Modelagem Matemática, Universidade Federal de Pelotas \\ Pelotas-RS, Brazil \\ ${ }^{2}$ Instituto de Física e Matemática, Universidade Federal de Pelotas \\ Pelotas-RS, Brazil \\ ${ }^{3}$ Instituto de Investigaciones en Matemáticas Aplicadas y Sistemas, Universidad Nacional Autónoma \\ de México \\ Ciudad de México, Mexico \\ roberto.decio@ufpel.edu.br,leslie.fernandez@ufpel.edu.br, julian@mym.iimas.unam.mx
}

Received: 19 April 2019; Accepted: 29 August 2019

\begin{abstract}
In their usual form, homogenization methods produce first-order approximations of the exact solutions of problems for differential equations with rapidly oscillating coefficients which model the physical behavior of microstructured media. However, there is need of approximations containing higher-order terms when the usual first-order approximations, which are formed by superposing a macroscopic trend and a local perturbation, are not capable of reproducing the local details of the exact solutions. Here, two-scale asymptotic solutions with second-order terms are provided for a Dirichlet problem modeling the steady state of functionally-graded microperiodic nonlinear rods. The need of considering higherorder terms is illustrated through numerical examples for various power-law nonlinearities.
\end{abstract}

MSC 2010: 34B15, 35B27, $74 K 10$

Keywords: asymptotic homogenization, power-law nonlinearity, functionally-graded rods

\section{Introduction}

Typical microstructured media are characterized by both separation of structural scales and the validity of the continuum hypothesis. This means that the characteristic length of the atomic/molecular scale is much smaller than the length scale of the assumed microstructural continuum of matter, which in turn is much smaller than the length scale of the apparent macroscopic homogeneity of matter. This implies that the hypothesis of equivalent homogeneity is valid, so the microstructured media behave as ideally homogeneous media whose physical properties are the so-called effective properties of the former $[1,2]$. Effective properties are the macroscopic magnitudes usually measured in experiments, contain all the microstructural and constitutive information of the real media, and do not depend on the position at the microscale [3]. 
The process of obtaining equivalent homogeneous media is called homogenization. From the mathematical point of view, the problems modeling the microstructured media have differential equations with microstructurally-induced rapidly oscillating coefficients, whereas the problems for the equivalent homogeneous media have equations with coefficients that are constant with respect to the microstructure. Then, the validity of the equivalent homogeneity hypothesis follows whenever the difference between the solutions of the problems for the microstructured and the homogeneous media is of the order of a power of the small geometrical parameter characterizing the separation of scales, with respect to an appropriate norm ${ }^{1}$ [1]. On the other hand, homogenization methods (such as: asymptotic homogenization [4,5]; two-scale convergence [6,7]; $\Sigma$-, $G$-, $\Gamma$ - and $H$-convergences [8-11]; oscillating test functions [12]; tangent second-order ${ }^{2}$ homogenization $[14,15]$; two-space homogenization $\left.[16,17]\right)$ usually produce only first-order ${ }^{3}$ approximations of the exact solutions of the problems that model the physical behavior of microstructured media. Such first-order approximations are formed by superposing a macroscopic trend and a local perturbation, which are related to the effective behavior and the influence of the microstructure, respectively. However, there is a need to consider approximations containing higher-order terms ${ }^{4}$ when knowledge of the details of the local behavior of the exact solutions is required and the traditional first-order approximations fail to reproduce such local details [18-20]. To the best of our knowledge, only asymptotic and twospace homogenization methods are capable of providing such higher-order terms. Also, such a situation is yet to be addressed in a nonlinear context. Here, asymptotic solutions containing second-order terms are provided via asymptotic homogenization for a two-point Dirichlet problem that models the steady state of functionally-graded microperiodic nonlinear rods. The need of considering higher-order terms is illustrated through numerical examples for various power-law nonlinearities.

\section{Theory}

Let $\varepsilon \in\left(0, \varepsilon_{0}\right), \varepsilon_{0} \ll 1$, be the small geometric parameter characterizing the separation of structural scales of a functionally-graded microperiodic cylindrical rod of negligible radius, which is constitutively nonlinear and whose steady state is modeled via the following two-point Dirichlet problem:

Original problem: For each small $\varepsilon>0$, find $u^{\varepsilon} \in C^{2}([0,1])$ such that

$$
\frac{d}{d x}\left[\sigma^{\varepsilon}\left(x, \frac{d u^{\varepsilon}}{d x}\right)\right]=f^{\varepsilon}(x), \quad u^{\varepsilon}(0)=a, \quad u^{\varepsilon}(1)=b,
$$

\footnotetext{
1 That is, a norm of the function space in which the solutions are sought.

2 It refers to approximations of the effective energy density which are exact up to the second order with respect to the contrast of the phase properties of composite media [13].

3 That is, approximations of the exact solution which are exact up to the first order with respect to the small geometrical parameter characterizing the separation of scales in microstructured media.

4 These approximations are still exact up to the first order in the small geometric parameter.
} 
where $\sigma^{\varepsilon}\left(x, \gamma^{\varepsilon}(x)\right)=\sigma\left(x, x / \varepsilon, \gamma^{\varepsilon}(x)\right)$ is the flux-type function which is continuously differentiable with respect to the spatial variable $x \in[0,1], \varepsilon$-periodic in $x$ in the second argument, and nonlinear and continuously differentiable with respect to the continuously differentiable gradient-type function $\gamma^{\varepsilon}(x)=d u^{\varepsilon} / d x$, and $f^{\varepsilon}(x)=f(x, x / \varepsilon)$ is a continuous distribution of point sources that is $\varepsilon$-periodic in the second argument. ${ }^{5}$ Such conditions guarantee the existence and uniqueness of solution $u^{\varepsilon}$ of problem (1) (see, Theorem 2.1, p. 92, of [22]).

Let $y=x / \varepsilon \in\left[0, \varepsilon^{-1}\right]$ be the so-called local variable and let $u_{k}(x, y), k \in\{0,1,2\}$, be certain unknown functions assumed to be twice continuously differentiable with respect to both arguments and 1-periodic with respect to $y$. Then, the formalism of asymptotic homogenization seeks a two-scale asymptotic solution of problem (1) as

$$
u^{\varepsilon}(x) \sim u^{(2)}(x, \varepsilon)=u_{0}(x, y)+\varepsilon u_{1}(x, y)+\varepsilon^{2} u_{2}(x, y)+O\left(\varepsilon^{3}\right) .
$$

Substitution of (2) into problem (1) uses the chain rule $d / d x=\partial / \partial x+\varepsilon^{-1} \partial / \partial y$, so, for $y=x / \varepsilon$, the gradient-type function $\gamma^{\varepsilon}$ is asymptotically approximated as

$$
\gamma^{\varepsilon}(x) \sim \frac{d u^{(2)}}{d x}=\varepsilon^{-1} \frac{\partial u_{0}}{\partial y}+\varepsilon^{0}\left(\frac{\partial u_{0}}{\partial x}+\frac{\partial u_{1}}{\partial y}\right)+\varepsilon\left(\frac{\partial u_{1}}{\partial x}+\frac{\partial u_{2}}{\partial y}\right)+O\left(\varepsilon^{2}\right) .
$$

Then, the asymptotic approximation of the flux-type function $\sigma^{\varepsilon}\left(x, \gamma^{\varepsilon}(x)\right)$ follows by substituting (3) into its Taylor linearization with respect to the gradient-type function $\gamma^{\varepsilon}$ with center $\gamma_{0}(x, y)$ for $\gamma_{j}(x, y)=\partial u_{j} / \partial x+\partial u_{j+1} / \partial y, j \in\{0,1\}$, to yield

$$
\begin{aligned}
\sigma^{\varepsilon}\left(x, \gamma^{\varepsilon}(x)\right) & \sim \varepsilon^{-1} \frac{\partial u_{0}}{\partial y} \frac{\partial \sigma}{\partial \gamma}\left(x, y, \gamma_{0}(x, y)\right)+\varepsilon^{0} \sigma\left(x, y, \gamma_{0}(x, y)\right) \\
& +\varepsilon \gamma_{1}(x, y) \frac{\partial \sigma}{\partial \gamma}\left(x, y, \gamma_{0}(x, y)\right)+O\left(\varepsilon^{2}\right) .
\end{aligned}
$$

Therefore, the equation of problem (1) is asymptotically approximated as

$$
\begin{gathered}
\frac{d}{d x}\left[\sigma^{\varepsilon}\left(x, \frac{d u^{(2)}}{d x}\right)\right]-f^{\varepsilon}(x) \sim \varepsilon^{-2} L_{y y} u_{0}+\varepsilon^{-1}\left\{L_{x y} u_{0}+\frac{\partial}{\partial y}\left[\sigma\left(x, y, \gamma_{0}(x, y)\right)\right]\right\} \\
+\varepsilon^{0}\left\{L_{y x} u_{1}+L_{y y} u_{2}+\frac{\partial}{\partial x}\left[\sigma\left(x, y, \gamma_{0}(x, y)\right)\right]-f(x, y)\right\}+O(\varepsilon),
\end{gathered}
$$

with the differential operator $L_{\alpha \beta}=(\partial / \partial \alpha)(\kappa(x, y) \partial / \partial \beta), \alpha, \beta \in\{x, y\}$, and the gradient-dependent diffusion-type ${ }^{6}$ property $\kappa(x, y)=(\partial \sigma / \partial \gamma)\left(y, \gamma_{0}(x, y)\right)$ which, for each fixed $\gamma_{0}(x, y)$, puts the information on the constitutive nonlinearity into the otherwise linear operator $L_{\alpha \beta}$.

\footnotetext{
${ }^{5}$ For various physical interpretations of magnitudes $u^{\varepsilon}, \gamma^{\varepsilon}$ and $\sigma^{\varepsilon}$ in the mechanical, electrical, magnetic and thermal contexts, see, for instance [2,21].

${ }^{6}$ Other interpretations of the material property $\kappa$ are, for instance, the strain-dependent secant modulus in nonlinear mechanics [13], and the electric field-dependent dielectric property in nonlinear electrostatics [23].
} 
The asymptotic equality in (5) implies that the asymptotic solution (2) solves the equation of problem (1) exactly up to the first order in $\varepsilon$ when there exist functions $u_{k}(x, y), k \in\{0,1,2\}$, which are twice continuously differentiable with respect to both arguments and 1-periodic with respect to $y$ and that nullify the coefficients of the nonpositive powers of $\varepsilon$ in (5). Then, by equating the coefficients of the nonpositive powers of $\varepsilon$ in (5) to zero, a recurrent sequence of problems is obtained for $u_{k}(x, y), k \in\{0,1,2\}$, with uniqueness conditions obtained by substituting (2) into the boundary conditions of problem (1) and taking their 1-periodicity in $y$ into account.

Problem from $O\left(\varepsilon^{-2}\right)$ term: For each $x \in[0,1]$, find $u_{0} 1$-periodic in $y$ such that

$$
L_{y y} u_{0}=0, \quad u_{0}(x, 0)=0 .
$$

Problem from $O\left(\varepsilon^{-1}\right)$ term: Let $u_{0}$ be the solution of problem (6). For each $x \in[0,1]$, find $u_{1} 1$-periodic in $y$ such that

$$
\frac{\partial}{\partial y}\left[\sigma\left(x, y, \frac{\partial u_{0}}{\partial x}+\frac{\partial u_{1}}{\partial y}\right)\right]=-L_{x y} u_{0}, \quad u_{1}(x, 0)=0 .
$$

Problem from $O\left(\varepsilon^{0}\right)$ term: Let $u_{j}, j \in\{0,1\}$, be the solutions of problems (6) and (7), respectively. For each $x \in[0,1]$, find $u_{2}$ 1-periodic in $y$ such that

$$
L_{y y} u_{2}=f(x, y)-\frac{\partial}{\partial x}\left[\sigma\left(x, y, \frac{\partial u_{0}}{\partial x}+\frac{\partial u_{1}}{\partial y}\right)\right]-L_{y x} u_{1}, \quad u_{2}(x, 0)=0 .
$$

The following lemma provides a necessary and sufficient condition for the existence and uniqueness of solutions of problems (6) and (8).

Lemma 1 [4]: Let $k(y)>0$ and $F(y)$ be 1-periodic functions with $k(y)$ bounded and continuously differentiable. Then, there exists a 1-periodic solution $N(y)$ of $L N \equiv$ $(d / d y)(k(y) d N / d y)=F(y)$ if and only if the mean value of $F(y)$ over the local period is null, that is $\langle F(y)\rangle \equiv \int_{0}^{1} F(y) d y=0$, where the angular brackets represent such a local mean value. Such a solution $N(y)$ is unique up to an additive constant $C$, that is, $N(y)=\tilde{N}(y)+C$, where $\tilde{N}(y)$ is the 1-periodic solution of $L N=F$ that satisfies the condition $\tilde{N}(0)=0$.

Proof: See, pp. 19-21, of [4].

For each $x \in[0,1]$, identify $k(y)=\kappa(x, y), L=L_{y y}, N(y)=u_{0}(x, y)$ and $F(y)=0$, so Lemma 1 applied to problem (6) implies that $u_{0}$ does not depend on $y, u_{0}(x, y)=$ $=v_{0}(x)$, so the first term $v_{0}$ of the asymptotic solution (2) is the macroscopic trend of the first-order approximation of the exact solution $u^{\varepsilon}$ of problem (1). The relation of $v_{0}(x)$ to the effective behavior is discussed later. Then, problems (7) and (8) become the so-called first and second local problems to find $u_{i}, i \in\{1,2\}$, respectively:

First local problem: Let $d v_{0} / d x$ be a parameter. For each $x \in[0,1]$, find $u_{1}$ 1-periodic in $y$ such that

$$
\frac{\partial}{\partial y}\left[\sigma\left(x, y, \frac{d v_{0}}{d x}+\frac{\partial u_{1}}{\partial y}\right)\right]=0, \quad u_{1}(x, 0)=0 .
$$


Second local problem: Let $d v_{0} / d x$ be a parameter. Let $u_{1}$ be the solution of problem (9). For each $x \in[0,1]$, find $u_{2} 1$-periodic in $y$ such that

$$
L_{y y} u_{2}=f(x, y)-\frac{\partial}{\partial x}\left[\sigma\left(x, y, \frac{d v_{0}}{d x}+\frac{\partial u_{1}}{\partial y}\right)\right]-L_{y x} u_{1}, \quad u_{2}(x, 0)=0 .
$$

Observe that, as $d v_{0} / d x$ is considered a parameter, both (9) and (10) can be regarded as parametric families of problems, and its correct choice is discussed later.

The following lemma guarantees the existence and uniqueness of the solution $u_{1}$ of the first local problem (9).

Lemma 2 [4]: Let $\bar{\gamma}$ be a parameter. Let $\gamma(y)$ be a continuously differentiable and 1-periodic function. Let $\sigma(y, \gamma(y))$ be continuously differentiable in both arguments and also 1-periodic in $y$. Then, there exist functions $N(y, \bar{\gamma})$ which are 1-periodic in $y$ and solve the parametric family of equations $(\partial / \partial y) \sigma(y, \bar{\gamma}+\partial N / \partial y)=0$ with parameter $\bar{\gamma}$. Such solutions $N(y, \bar{\gamma})$ are unique up to an additive constant $C$, that is, $N(y, \bar{\gamma})=\tilde{N}(y, \bar{\gamma})+C$, where $\tilde{N}(y, \bar{\gamma})$ are the 1-periodic solutions that satisfy the condition $\tilde{N}(0, \bar{\gamma})=0$.

Proof: Observe that $\sigma(y, \gamma(y))$ does not depend on $y$ for $\gamma(y)=\bar{\gamma}+\partial N / \partial y$, that is, $\sigma(y, \bar{\gamma}+\partial N / \partial y)=\langle\sigma(y, \bar{\gamma}+\partial N / \partial y)\rangle=\bar{\sigma}$ for some flux-type magnitude $\bar{\sigma}$ that does not depend on $y$. Then, application of the implicit function theorem produces $\gamma(y, \bar{\sigma})=\bar{\gamma}+\partial N / \partial y$, which denotes the inverse function of $\sigma(y, \bar{\gamma}+\partial N / \partial y)$ with respect to the second argument, and that is 1-periodic in $y$. Therefore, it follows that $N(y, \bar{\gamma})$ is the primitive of $\gamma(y, \bar{\sigma})-\bar{\gamma}$ and whose 1-periodicity in $y$ guaranteed as $\langle\gamma(y, \bar{\sigma})\rangle=\bar{\gamma}$

For each $x \in[0,1]$, by identifying $\bar{\gamma}=d v_{0} / d x$ and $N(y, \bar{\gamma})=u_{1}(x, y)$, application of Lemma 2 to the first local problem (9) also implies that there exists the so-called effective flux-type function $\hat{\sigma}\left(x, d v_{0} / d x\right) \equiv\left\langle\sigma\left(x, y, d v_{0} / d x+\partial u_{1} / \partial y\right)\right\rangle$, in which $u_{1}$ is the solution of problem (9) and provides the local perturbation to the macroscopic trend $v_{0}$. Furthermore, the relation $\bar{\sigma}=\hat{\sigma}(x, \bar{\gamma})$ is the so-called effective law relating the macroscopic mean flux-type and gradient-type variables $\bar{\sigma}$ and $\bar{\gamma}$ which describes the effective nonlinear behavior of the microstructured rod. Interestingly, obtaining such an effective law, which is sufficient for many applications, does not require explicit knowledge of the solution $u_{1}$ of the first local problem (9) but only its derivative with respect to $y$.

On the other hand, for each $x \in[0,1]$, by identifying $k(y)=\kappa(x, y), L=L_{y y}$, $N(y)=u_{2}(x, y)$ and $F(y)=f(x, y)-(\partial / \partial x)\left[\sigma\left(y, \partial u_{0} / \partial x+\partial u_{1} / \partial y\right)\right]-L_{y x} u_{1}$, and observing that $\left\langle L_{y x} u_{1}\right\rangle=0$, application of Lemma 1 to the second local problem (10) implies that the existence of its solution $u_{2}$ depends on the existence of the solution $v_{0}$ of the so-called homogenized problem:

Homogenized problem: For $\hat{f}(x)=\langle f(x, y)\rangle$, find $v_{0} \in C^{2}([0,1])$ such that

$$
\frac{d}{d x}\left[\hat{\sigma}\left(x, \frac{d v_{0}}{d x}\right)\right]=\hat{f}(x), \quad v_{0}(0)=a, \quad v_{0}(1)=b .
$$


Let $v_{0}$ and $u_{i}, i \in\{1,2\}$, be the solutions of problems (11), (9) and (10), respectively. Then, the asymptotic solution $u^{(2)}$ from (2) becomes

$$
u^{\varepsilon}(x) \sim u^{(2)}(x, \varepsilon)=v_{0}(x)+\varepsilon u_{1}\left(x, \frac{x}{\varepsilon}\right)+\varepsilon^{2} u_{2}\left(x, \frac{x}{\varepsilon}\right)+O\left(\varepsilon^{3}\right),
$$

which, in view of the asymptotic equality (5), approximates the exact solution $u^{\varepsilon}$ of the original problem (1) exactly up to the first order in $\varepsilon$, that is,

$$
\left\|\frac{d}{d x}\left[\sigma\left(x, \frac{x}{\varepsilon}, \frac{d u^{(2)}}{d x}\right)\right]-f\left(x, \frac{x}{\varepsilon}\right)\right\|_{C([0,1])}=O(\varepsilon),
$$

so the second-order term $\varepsilon^{2} u_{2}$ appears to be useful only for obtaining the homogenized problem (11) for the first term $v_{0}$ of the asymptotic solution (12). For this reason, most applications do not consider (and most methods are not capable of obtaining) such a second-order term, but are rather limited to the asymptotic solution $u^{(1)}$ given by

$$
u^{\varepsilon}(x) \sim u^{(1)}(x, \varepsilon)=v_{0}(x)+\varepsilon u_{1}\left(x, \frac{x}{\varepsilon}\right)+O\left(\varepsilon^{2}\right),
$$

which considers only the terms contributing to the first-order exactness in $\varepsilon$ of the asymptotic approximation of the exact solution $u^{\varepsilon}$ of the original problem (1): the macroscopic trend $v_{0}$ related to the effective behavior via the flux-type function $\hat{\sigma}\left(x, d v_{0} / d x\right)$, and the local perturbation $\varepsilon u_{1}$ of order of the relative length scale $\varepsilon$ of the microstructure. In fact, approximations of the exact solution $u^{\varepsilon}$ similar to (12) are what the other homogenization methods produce, that is, a superposition of a macroscopic trend and a local perturbation. However, there are situations in which the contribution of such a second-order term $\varepsilon^{2} u_{2}$ must not be neglected. Specifically, the asymptotic solution $u^{(2)}$ in (12) must be considered when the asymptotic solution $u^{(1)}$ in (14) fails to reproduce the details of the local behavior of the exact solution $u^{\varepsilon}$ of the original problem (1). This fact is relevant as in most situations the exact solution $u^{\varepsilon}$ is not available. Therefore, if knowledge of the local details of the behavior of $u^{\varepsilon}$ is required, the alternative to the unavailability of $u^{\varepsilon}$ provided by the first-order asymptotic approximation must be as locally accurate as possible, so choosing $u^{(2)}$ over $u^{(1)}$ is the correct approach.

\section{Results}

Consider the original problem (1) for the source term $f^{\varepsilon}(x)=-1$, the boundary values $a=0$ and $b=1$, and the power-law flux-type function [13,24,25] given by

$$
\sigma^{\varepsilon}\left(x, \frac{d u^{\varepsilon}}{d x}\right)=k^{\varepsilon}(x)\left(\frac{d u^{\varepsilon}}{d x}\right)^{n}, \quad k^{\varepsilon}(x)=1+\frac{1}{4} \sin 2 \pi \frac{x}{\varepsilon} .
$$


Usual linear behavior corresponds to $n=1$, whereas the values of the nonlinearity exponent $n \in \mathbb{R}_{+}^{*} \backslash\{1\}$ considered here correspond to the so-called strongly nonlinear behavior for $0<n<1$ and to the so-called weakly nonlinear behavior for $n>1$, respectively. Also, the coefficient $k^{\varepsilon}$ is positive, bounded and $\varepsilon$-periodic, and oscillate rapidly for sufficiently small values of $\varepsilon$. With such considerations, the exact solution $u^{\varepsilon}$ of the original problem (1) is obtained via direct integration as

$$
u^{\varepsilon}(x)=\int_{0}^{x}\left(\frac{C^{\varepsilon}-s}{k^{\varepsilon}(s)}\right)^{1 / n} d s
$$

where $C^{\varepsilon}$ is a constant whose value is obtained by substituting the boundary condition $u^{\varepsilon}(1)=1$ into (16). Observe that $u^{\varepsilon}(x) \rightarrow x$ as $n \rightarrow \infty$, which is useful to control the computational results.

Figures 1 and 2 show the behavior of the exact solution $u^{\varepsilon}$ in (16) for various values of $\varepsilon$ and $n$. Figure 1 illustrates the fact that decreasing values of $\varepsilon$ indicate finer microstructures, so the finer the microstructure, the more rapidly $u^{\varepsilon}$ varies locally.

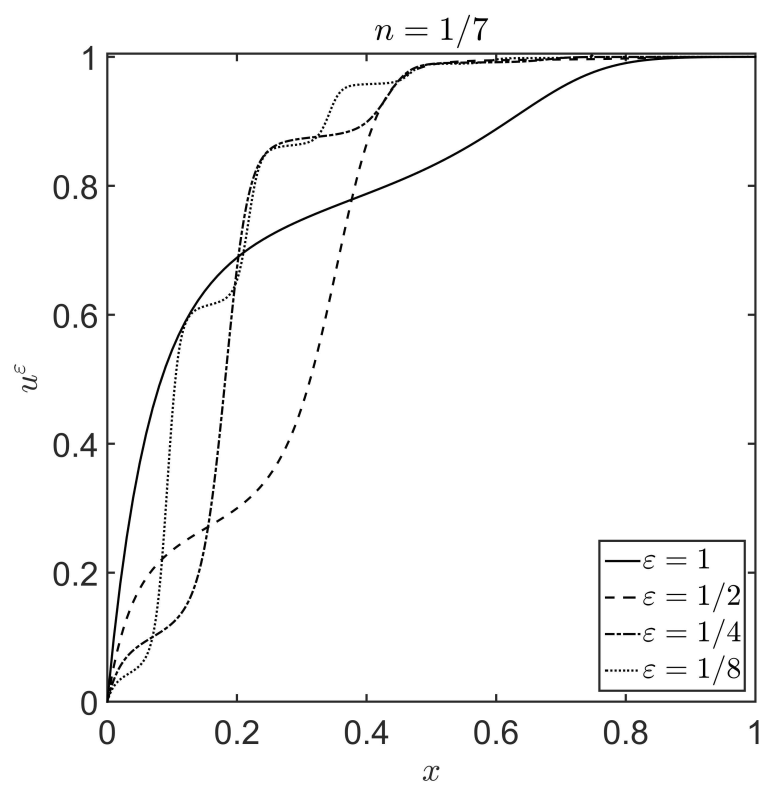

Fig. 1. Exact solution $u^{\varepsilon}$ in (16) for $n=1 / 7$ and various values of $\varepsilon$

On the other hand, Figure 2 shows that, as expected, $u^{\varepsilon}$ approaches the identity function for increasing values of $n$, that is, for weaker nonlinearities. Moreover, observe that weaker nonlinearities exhibit less local variability and, conversely, stronger nonlinearties exhibit more local variability. Therefore, it seems reasonable to expect that the asymptotic solution $u^{(1)}$ in (14) will be sufficient to approximate the exact solution $u^{\varepsilon}$ with good local accuracy for weak nonlinearities, whereas the asymptotic solution $u^{(2)}$ in (12) containing a second-order term will be necessary to approximate the exact solution $u^{\varepsilon}$ with good local accuracy for strong nonlinearities. 


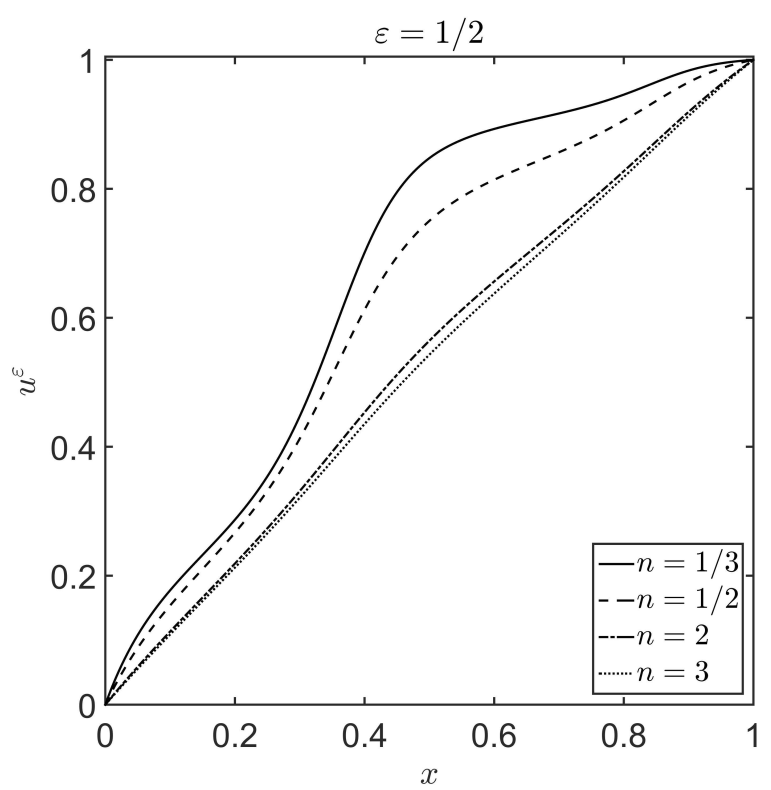

Fig. 2. Exact solution $u^{\varepsilon}$ in (16) for $\varepsilon=1 / 2$ and various values of $n$

The effective flux-type function also exhibits power-law behavior as

$$
\hat{\sigma}\left(\frac{d v_{0}}{d x}\right)=\hat{k}\left(\frac{d v_{0}}{d x}\right)^{n}, \quad \hat{k}=\left\langle k^{-1 / n}(y)\right\rangle^{-n},
$$

where $\hat{k}$ is the so-called effective coefficient. Then, the solution $v_{0}$ of the homogenized problem (11) corresponding to the effective flux-type function (17), the source term $\hat{f}(x)=-1$, and the boundary values $a=0$ and $b=1$, is

$$
v_{0}(x)=\hat{k}^{-1 / n}\left(C_{0}^{(n+1) / n}-\left(C_{0}-x\right)^{(n+1) / n}\right),
$$

where the value of $C_{0}$ is obtained by substituting the boundary condition $v_{0}(1)=1$ into (18). Observe that $v_{0}(x) \rightarrow x$ as $n \rightarrow \infty$, so the macroscopic trend $v_{0}$ seems to approximate the exact solution $u^{\varepsilon}$ with sufficient accuracy for weak nonlinearities.

The solution $u_{1}$ of the first local problem (9) corresponding to the flux-type function (15) is obtained following the ideas in the proof of Lema 2 as

$$
u_{1}\left(x, \frac{x}{\varepsilon}\right)=N_{1}^{\varepsilon}(x) \frac{d v_{0}}{d x}, \quad N_{1}(y)=\hat{k}^{1 / n} \int_{0}^{y} \frac{d s}{k^{1 / n}(s)}-y,
$$

for $y \in[0,1]$, so the so-called first local function $N_{1}^{\varepsilon}(x)=N_{1}(x / \varepsilon)$ is defined over the period and must be extended to be $\varepsilon$-periodic. Observe that, $u_{1} \rightarrow 0$ as $n \rightarrow \infty$, that is, the first-order local perturbation becomes negligible for weak nonlinearities, which reassures the idea that the macroscopic trend $v_{0}$ appears to be a sufficiently accurate approximation of the exact solution $u^{\varepsilon}$ for sufficiently weak nonlinearities. 
The solution $u_{2}$ of the second local problem (10) corresponding to the flux-type function (15) is obtained as

$$
u_{2}\left(x, \frac{x}{\varepsilon}\right)=N_{2}^{\varepsilon}(x) \frac{d^{2} v_{0}}{d x^{2}}, \quad N_{2}(y)=\left\langle N_{1}(y)\right\rangle\left(N_{1}(y)+y\right)-\int_{0}^{y} N_{1}(s) d s
$$

also for $y \in[0,1]$ and with $N_{1}(y)$ from (19), so the so-called second local function $N_{2}^{\varepsilon}(x)=N_{2}(x / \varepsilon)$ is defined over the period and must be extended to be $\varepsilon$-periodic. Observe that, $u_{2} \rightarrow 0$ as $n \rightarrow \infty$, that is, the second-order local perturbation also becomes negligible for sufficiently weak nonlinearities, which reassures the idea that the macroscopic trend $v_{0}$ appears to be a sufficiently accurate asymptotic approximation of the exact solution $u^{\varepsilon}$ for sufficiently weak nonlinearities.

Figures 3 and 4 show the local functions $N_{1}^{\varepsilon}$ and $N_{2}^{\varepsilon}$ in (19) and (20), respectively, for various values of $\varepsilon$ and $n$. Figure 3 shows the behavior of the first local function $N_{1}^{\varepsilon}$ and illustrates the fact that decreasing values of $\varepsilon$ indicate finer microstructures, so the finer the microstructure, the more rapidly $N_{1}^{\varepsilon}$ varies locally. The second local function $N_{2}^{\varepsilon}$ behaves similarly for decreasing values of $\varepsilon$.

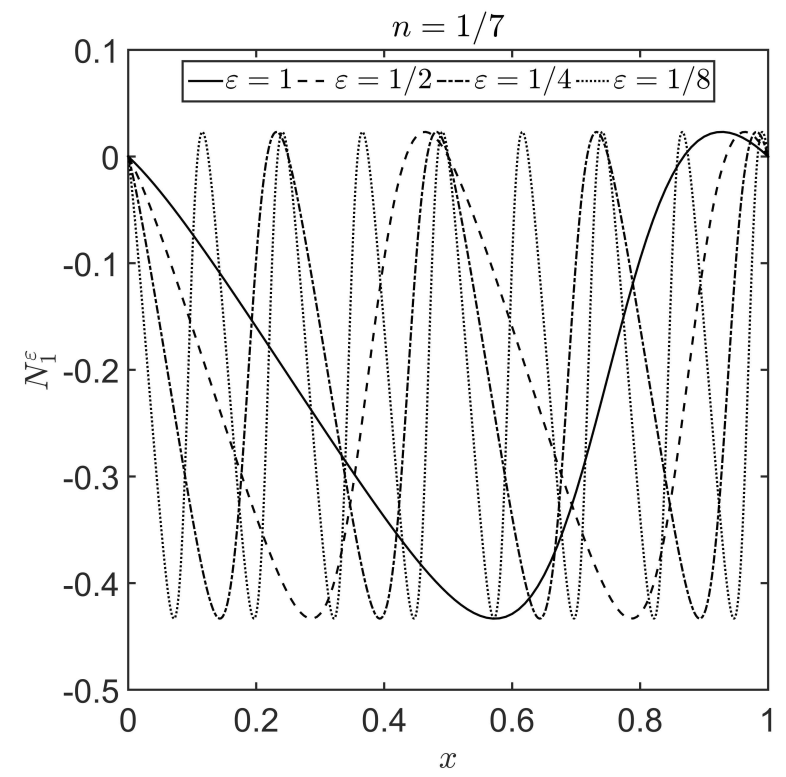

Fig. 3. First local function $N_{1}^{\varepsilon}$ in (19) for $n=1 / 7$ and various values of $\varepsilon$

On the other hand, Figure 4 shows the behavior of the second local function $N_{2}^{\varepsilon}$ and illustrates the fact that increasing values of $n$ diminishes the influence of the microstructure, as the amplitude of the oscillations of $N_{2}^{\varepsilon}$ decreases. The first local function $N_{1}^{\varepsilon}$ behaves similarly for increasing values of $n$. Therefore, both first- and second-order local perturbations become negligible for sufficiently weak nonlinearities, which reassures the idea that the macroscopic trend $v_{0}$ appears to be a sufficiently accurate approximation of the exact solution $u^{\varepsilon}$ for sufficiently weak nonlinearities. 


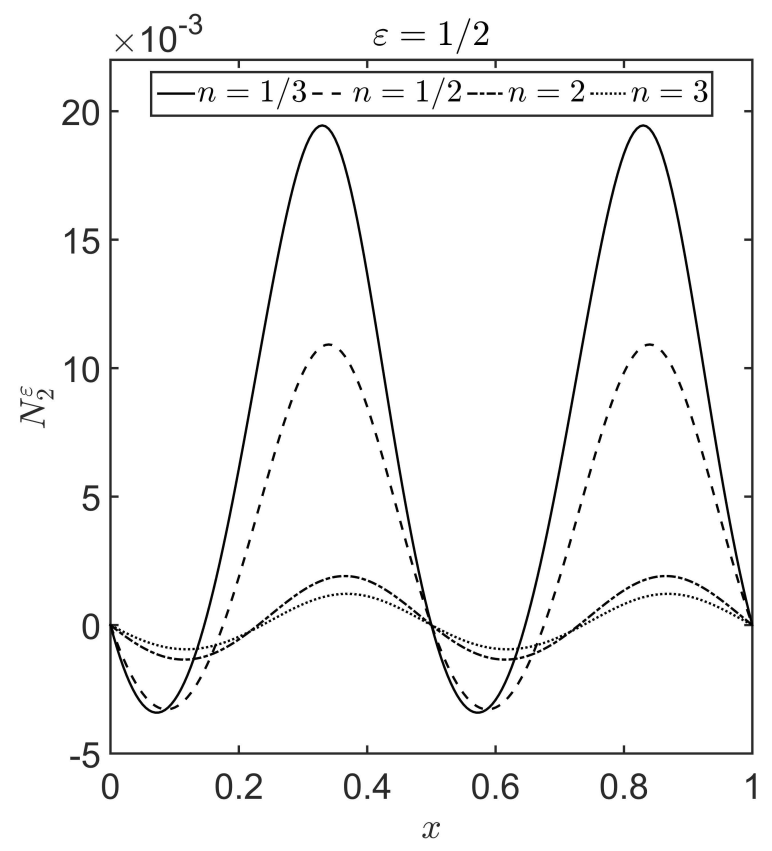

Fig. 4. Second local function $N_{2}^{\varepsilon}$ in (20) for $\varepsilon=1 / 2$ and various values of $n$

The asymptotic solutions $u^{(1)}$ and $u^{(2)}$ containing up to first- and second-order terms in $\varepsilon$, respectively, which approximate the exact solution $u^{\varepsilon}$ in (16), follow by substituting (18) and (19) into (14) and (18)-(20) into (12), respectively. Recall that it is expected that the asymptotic solution $u^{(1)}$ will be sufficient to approximate the exact solution $u^{\varepsilon}$ with good local accuracy for weakly nonlinear behaviors, whereas the asymptotic solution $u^{(2)}$ containing a second-order term will be necessary to approximate the exact solution $u^{\varepsilon}$ with good local accuracy for strongly nonlinear behaviors and, also, that the macroscopic trend $v_{0}$ will be sufficient to approximate the exact solution $u^{\varepsilon}$ for sufficiently weak nonlinear behaviors. In order to evaluate such expectations, the quality of the asymptotic approximations of the exact solution $u^{\varepsilon}$ provided by $v_{0}, u^{(1)}$ and $u^{(2)}$ is quantified via the root-mean-square error (as given by the discrete version of the $L^{2}([0,1])$-norm) of such approximations with respect to the exact solution.

Table 1. Quality of the approximations $v_{0}, u^{(1)}$ and $u^{(2)}$ of $u^{\varepsilon}$ for $\varepsilon=1 / 2$

\begin{tabular}{cccc}
\hline$n$ & $\left\|u^{\varepsilon}-v_{0}\right\|_{L^{2}([0,1])}$ & $\left\|u^{\varepsilon}-u^{(1)}\right\|_{L^{2}([0,1])}$ & $\left\|u^{\varepsilon}-u^{(2)}\right\|_{L^{2}([0,1])}$ \\
\hline $1 / 7$ & $2.2275 \cdot 10^{-1}$ & $1.0162 \cdot 10^{-1}$ & $3.0656 \cdot 10^{-2}$ \\
$1 / 5$ & $1.6311 \cdot 10^{-1}$ & $4.8664 \cdot 10^{-2}$ & $8.7127 \cdot 10^{-3}$ \\
$1 / 3$ & $8.9069 \cdot 10^{-2}$ & $1.2667 \cdot 10^{-2}$ & $5.2093 \cdot 10^{-3}$ \\
1 & $2.3395 \cdot 10^{-2}$ & $3.8951 \cdot 10^{-3}$ & $4.0772 \cdot 10^{-3}$ \\
3 & $6.3654 \cdot 10^{-3}$ & $4.0326 \cdot 10^{-3}$ & $4.0508 \cdot 10^{-3}$ \\
5 & $3.7614 \cdot 10^{-3}$ & $4.0510 \cdot 10^{-3}$ & $4.0565 \cdot 10^{-3}$ \\
7 & $3.1245 \cdot 10^{-3}$ & $4.0562 \cdot 10^{-3}$ & $4.0588 \cdot 10^{-3}$ \\
\hline
\end{tabular}


Table 2. Quality of the approximations $v_{0}, u^{(1)}$ and $u^{(2)}$ of $u^{\varepsilon}$ for $\varepsilon=1 / 16$

\begin{tabular}{cccc}
\hline$n$ & $\left\|u^{\varepsilon}-v_{0}\right\|_{L^{2}([0,1])}$ & $\left\|u^{\varepsilon}-u^{(1)}\right\|_{L^{2}([0,1])}$ & $\left\|u^{\varepsilon}-u^{(2)}\right\|_{L^{2}([0,1])}$ \\
\hline $1 / 7$ & $3.2384 \cdot 10^{-2}$ & $2.2893 \cdot 10^{-3}$ & $1.2652 \cdot 10^{-3}$ \\
$1 / 5$ & $2.1678 \cdot 10^{-2}$ & $8.7342 \cdot 10^{-4}$ & $1.0129 \cdot 10^{-3}$ \\
$1 / 3$ & $1.1233 \cdot 10^{-2}$ & $5.8235 \cdot 10^{-4}$ & $7.3442 \cdot 10^{-4}$ \\
1 & $2.9003 \cdot 10^{-3}$ & $5.3045 \cdot 10^{-4}$ & $5.3734 \cdot 10^{-4}$ \\
3 & $7.9377 \cdot 10^{-4}$ & $5.1273 \cdot 10^{-4}$ & $5.1298 \cdot 10^{-4}$ \\
5 & $4.7247 \cdot 10^{-4}$ & $5.1101 \cdot 10^{-4}$ & $5.1106 \cdot 10^{-4}$ \\
7 & $3.9400 \cdot 10^{-4}$ & $5.1051 \cdot 10^{-4}$ & $5.1053 \cdot 10^{-4}$ \\
\hline
\end{tabular}

Tables 1 and 2 present such a quality quantification of the three asymptotic approximations $v_{0}, u^{(1)}$ and $u^{(2)}$ of the exact solution $u^{\varepsilon}$ for $\varepsilon=1 / 2$ and $\varepsilon=1 / 16$, respectively, and various values of $n$. Remarkably, all expectations were met, that is, the errors for the three approximations were of the same order for weak nonlinearities $(n>1)$ being $\sim 10^{-3}$ and $\sim 10^{-4}$ for $\varepsilon=1 / 2$ and $\varepsilon=1 / 16$, respectively, whereas the error decreases by around one order of magnitude when considering the approximation containing one more higher-order term in $\varepsilon$ than the previous for strong nonlinearities $(n<1)$.

\section{Conclusions}

Here, the asymptotic homogenization method was applied to a two-point Dirichlet problem modeling the steady state of functionally-graded microperiodic nonlinear rods. Two-scale asymptotic solutions with second-order terms are obtained to account for the cases in which the usual first-order approximations, that are formed by superposing a macroscopic trend and a local perturbation, are not capable of reproducing the local details of the exact solutions determined by the microperiodicity. It was found that, when considering power-law nonlinearities, the asymptotic solution containing up to first-order terms is sufficient to approximate the exact solution of the problem with good local accuracy for weakly nonlinear behaviors, whereas the asymptotic solution containing up to second-order terms is necessary to approximate the exact solution of the problem with good local accuracy for strongly nonlinear behaviors. Various numerical examples were performed and confirmed these findings.

\section{References}

[1] Panasenko, G.P. (2008). Homogenization for periodic media: from microscale to macroscale. Physics of Atomic Nuclei, 71(4), 681-694.

[2] Torquato, S. (2002). Random Heterogeneous Materials: Microstructure and Macroscopic Properties. Springer.

[3] Christensen, R.M. (1979). Mechanics of Composite Materials. Wiley.

[4] Bakhvalov, N.S., \& Panasenko, G.P. (1989). Homogenisation: Averaging Processes in Periodic Media. Kluwer. 
[5] Bensoussan, A., Lions, J.-L., \& Papanicolau, G. (1978). Asymptotic Analysis for Periodic Structures. North-Holland.

[6] Allaire, G. (1992). Homogenization and two-scale convergence. SIAM Journal on Mathematical Analysis, 23(6), 1482-1518.

[7] Nguetseng, G. (1989). A general convergence result for a functional related to the theory of homogenization. SIAM Journal on Mathematical Analysis, 20(3), 608-623.

[8] Nguetseng, G. (2003). Homogenization structures and applications I. Zeitschrift für Analysis und ihre Anwendungen, 22(1), 73-108.

[9] Spagnolo, S. (1976). Convergence in energy for elliptic operators. In: Numerical Solution of Partial Differential Equations (Vol. III). Academic Press, 468-498.

[10] De Giorgi, E. (1984). G-operators and $\Gamma$-convergence. In: Proceedings of the International Congress of Mathematicians (Vol. 2). North-Holland, 1175-1191.

[11] Murat, F., \& Tartar, L. (1995). H-convergence. In: Topics in the Mathematical Modeling of Composite Materials: Progress in Nonlinear Differential Equations and Their Applications (Vol. 31). Springer, 21-43.

[12] Tartar, L. (2010). The General Theory of Homogenization: A Personalized Introduction. Springer.

[13] Ponte Castañeda, P., \& Suquet, P. (1998). Nonlinear composites. Advances in Applied Mechanics, 34, 171-302.

[14] Ponte Castañeda, P. (2002). Second-order homogenization estimates for nonlinear composites incorporating field fluctuations: I-theory. Journal of the Mechanics and Physics of Solids, 50(4), 737-757.

[15] Ponte Castañeda, P., \& Tiberio, E. (2000). A second-order homogenization method in finite elasticity and applications to black-filled elastomers. Journal of the Mechanics and Physics of Solids, 48(6-7), 1389-1411.

[16] Keller, J.B. (1977). Effective behavior of heterogeneous media. In: Statistical Mechanics and Statistical Methods in Theory and Applications, Plenum, 631-644.

[17] Keller, J.B. (1980). Darcy's law for flow in porous media and the two-space method. In: Nonlinear Partial Equations in Engineering and Applied Science: Lecture Notes in Pure and Applied Mathematics (Vol. 54), Dekker, 429-443.

[18] Su, F., Xu, Z., Cui, J.Z., \& Dong, Q.L. (2011). Multi-scale method for the quasi-periodic structures of composite materials. Applied Mathematics and Computation, 217(12), 5847-5852.

[19] Su, F., Xu, Z., Dong, Q.L., \& Jiang, H. (2011). Multiscale computation method for parabolic problems of composite materials. Applied Mathematics and Computation, 217(21), 8337-8342.

[20] Dong, Q.L., \& Cao, L.Q. (2014). Multiscale asymptotic expansions methods and numerical algorithms for the wave equations in perforated domains. Applied Mathematics and Computation, 232, 872-887.

[21] Hashin, Z. (1983). Analysis of composite materials - a survey. Journal of Applied Mechanics, 50, 481-505.

[22] Elsgolts, L. (1970). Differential Equations and the Calculus of Variations. Mir.

[23] Talbot, D.R.S., \& Willis, J.R. (1994). Upper and lower bounds for the overall properties of a nonlinear composite dielectric. I. Random microgeometry. Proceedings of the Royal Society of London A, 447, 365-384.

[24] Dai, G., Jarrar, F., Ozturk, F., \& Sheikh-Ahmad, J. (2018). On the effect of the complexity of the constitutive model in simulating superplastic forming. Defect and Diffusion Forum, 385, 379-384.

[25] Padmanabhan, K.A., Prabu, S.B., \& Ali, A.A. (2018). On the nuances in the power law description and interpretation of high homologous temperature creep and superplasticity data. Defect and Diffusion Forum, 385, 27-32. 